\title{
STRAIGHT CHERN CHARACTER FOR WITT SPACES
}

Henri MOSCOVICI and Fangbing WU
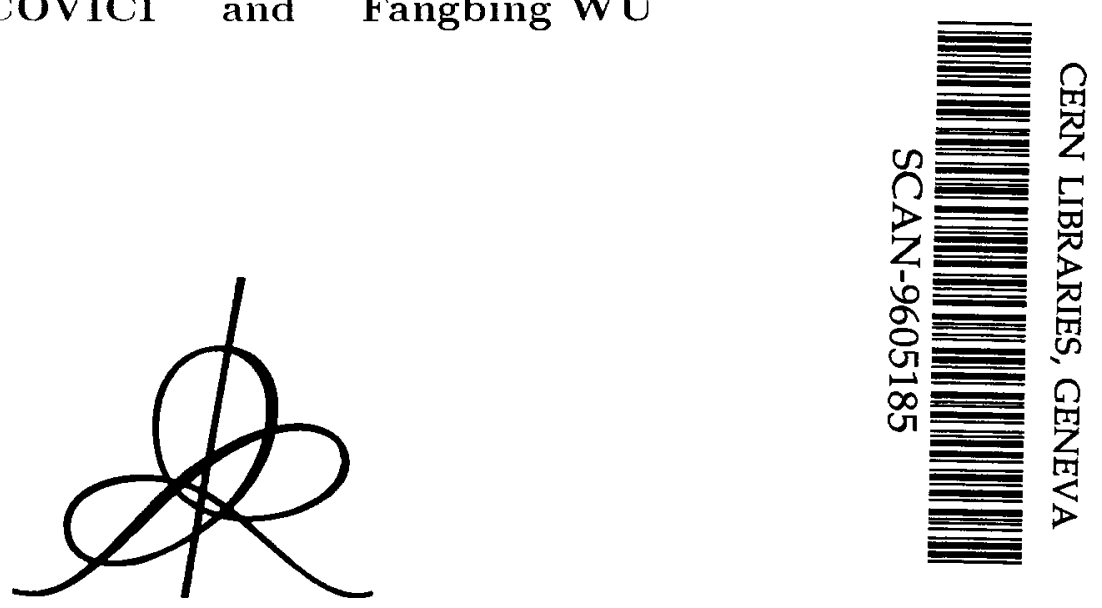

$\operatorname{swg} 623$

Institut des Hautes Etudes Scientifiques

35 , route de Chartres

91440 - Bures-sur-Yvette (France)

Mars 1996

IHES/M/96/21 


\title{
Straight Chern Character for Witt Spaces
}

\author{
Henri Moscovici * Fangbing Wu
}

\begin{abstract}
We show that the Goresky-MacPherson homology $\mathcal{L}$-class of a Witt space $X$ can be represented, in a local fashion, as the 'straight' Chern character of the Cheeger signature $K$-cycle constructed out of any piecewise Riemannian metric of conic type on $X$.
\end{abstract}

\section{Introduction}

The signature operator associated to a Riemannian metric on a closed smooth manifold $M$ defines a $K$-cycle over the $C^{*}$-algebra $C(M)$, which represents a fundamental (or $K$-orientation) $K$-homology class $\Delta_{M} \in K_{*}(M) \otimes \mathbb{Z}[1 / 2]$. Furthermore, its homological Chern character coincides, up to normalization, with the dual $L$-class of $M$. There is a remarkable extension of this fact to a natural class of $\mathrm{PL}$ 'manifolds with singularities', that satisfy a Poincaré duality property with rational coefficients, called Witt spaces. For such a space $X$, Siegel [Si] has constructed a class $\Delta_{X} \in K_{*}(X) \otimes \mathbb{Z}[1 / 2]$ which generalizes Sullivan's [Su] $K$-orientation class for PL manifolds. He also extended to Witt spaces the Goresky-MacPherson [GM] procedure of defining homology $L$-classes. On the other hand, Cheeger [C1] has shown that if $X$ is oriented and endowed with a piecewise smooth Riemannian metric of conic type, then one can canonically construct an essentially selfadjoint signature operator $\mathrm{D}$, acting on the Hilbert space $\mathcal{H}$ of $L^{2}$-forms on the regular part of $X$. This operator again defines a $K$-cycle, $(\mathcal{H}, \mathrm{D})$ over $C(X)$.

\footnotetext{
*J. S. Guggenheim Fellow

Research supported in part by the National Science Foundation
} 
In the special case when the metric on $X$ is chosen to be piecewise. qat, Cheeger [C2] has obtained local formulae, in terms of $\eta$-invariants of links, for the signature with coefficients in vector bundles equipped with partially flat connections. These yield local combinatorial expressions for the GoreskyMacPherson-Siegel $L$-classes of $X$ and also imply that the $K$-homology class with rational coefficients defined by the signature operator associated to a piecewise flat metric on $X$ is the same as the $K$-orientation class $\Delta_{X} \otimes \mathbb{Q}$.

Our goal here is to prove that the Goresky-MacPherson-Siegel homology $L$-classes of $X$ can still be represented in a local fashion starting from any piecewise smooth Riemannian metric of conic type. This is achieved by means of the 'straight' Chern character [CM] of the corresponding $K$-cycle $(\mathcal{H}, \mathrm{D})$, in a manner similar to the local representation of the topological Pontryagin classes in [MW2] (comp. also [CST]). As Alexander-Spanier cycles, these local expressions are less appealing than Cheeger's local combinatorial formulae, but they do not require the piecewise flatness assumption. It should also be noted that the more direct route we are taking here bypasses Cheeger's rather delicate analysis, using only its 'soft' and quasi-isometrically stable aspects, and thus is better positioned for generalization.

\section{Witt Spaces and Cheeger's Operators}

We briefly recall a few basic notions concerning Witt spaces, Riemannian structures with conic singularities and associated de Rham operators.

To begin with, a PL pseudomanifold of dimension $n$ is a PL space $X^{n}$ of dimension $n$ containing a closed PL space $\Sigma^{n-2}$ of codimension $\geq 2$ such that $X^{n} \backslash \Sigma^{n-2}$ is an $n$-dimensional PL manifold dense in $X^{n}$. The open dense subset $X^{n} \backslash \Sigma^{n-2}$ is called the regular part of $X$ and will also be denoted $X^{\text {reg }}$. Equivalently, for a compatible triangulation $T, X^{n}$ is the union of the closed $n$-simplices and each $n-1$-simplex is a face of either one or two $n$-simplices; a simplex which is the face of only one $n$-simplex is part of the boundary $\partial X^{n}$. The pseudomanifold $X^{\prime \prime}$ is oriented if its regular part is oriented. An orientation on $X^{n}$ is given by a coherent orientation of all the $n$-simplices of a compatible triangulation $T$ of $X^{n}$.

Any PL pseudomanifold $X^{n}$ admits a $P L$ stratification, i.e. a filtration by closed PL subspaces

$$
\emptyset=X_{-1} \subset X_{1} \subset \cdots \subset X_{n-3} \subset X_{n-2}=X_{n-1}=\Sigma^{n-2} \subset X^{n}
$$


such that

(i) $S_{i}:=X_{i} \backslash X_{i-1}$ is empty or an $i$-dimensional manifold called stratum;

(ii) for any $x \in S_{i}$, there is a neighborhood $U$ of $x$ in $S_{i}$, a compact stratified pseudomanifold $L_{x}$ and a stratum preserving $\mathrm{PL}$ embedding

$$
\varphi_{x}: U \times c\left(L_{x}\right) \longrightarrow X
$$

the last condition is called the local normal triviality property and the $L_{x}$ 's are called links.

Indeed, if $T$ is a compatible triangulation of pseudomanifold $X^{n}$ such that $\Sigma^{n-2}$ is a subcomplex, we can take $\mathrm{X}_{k}$ to be the union of the closed simplices of dimension $\leq k$ contained in $\Sigma^{n-2}$. The links corresponding to such a stratification by skeleta are the usual links of the simplices of $T$ with respect to the first barycentric subdivision.

The appropriate homological tools for the study of pseudomanifolds are the family of topological invariants introduced by Goresky and MacPherson $[\mathrm{GM}]$, called intersection homology groups. These family is parametrized by a collection of sequences of non-negative integers called perversities. In particular, the sequence $\{0,0,1,1,2,2, \ldots\}$ represents the middle perversity. We shall denote by $I H_{*}$ the intersection homology groups corresponding to the middle perversity.

A Witt space is an oriented PL pseudomanifold $X$ such that the (rational) middle perversity intersection homology groups of the links of all strata of odd codimension vanish in the middle dimension; that is

$$
I H_{\frac{l}{2}}\left(L_{x} ; \mathbb{Q}\right)=\{0\}, \quad \forall x \in X \text { with } l=\operatorname{dim}\left(L_{x}\right) \text { even. }
$$

This property is independent of the stratification (cf. [Si, Prop. 2.5]).

We now proceed to define, following (heeger [C1], a natural class of Riemannian structures with singularities on pseudomanifolds.

Assume first that the pseudomanifold $X^{n}$ is embedded as a subcomplex in some piecewise linear triangulation of $\mathbb{R}^{n}$. The induced metric on $X^{n}$ $\Sigma^{n-2}$ makes $X^{n} \backslash \Sigma^{n-2}$ a smooth flat Riemannian manifold. By definition, a piecewise Riemannian metric of conic type is a metric space structure on $X^{n}$ such that the induced metric on $X^{r e g}$ is piecewise smoothly quasi-isometric to the flat metric. 
Let us now fix such a metric $g$ on the pseudomanifold $X$. Denote br $\Lambda^{i}$ (resp. $\Lambda$ ) the space of smooth $i$-forms (resp. forms) on $X^{\text {reg }}$ with arbitrary supports, and by $\mathcal{H}_{g}$ be the Hilbert space of $L^{2}$-forms on the (incomplete) Riemannian manifold $\left(X^{r e g}, g\right)$. We consider $d$, the de Rham exterior derivation, as an unbounded operator with domain

$$
\operatorname{dom}(d):=\left\{\omega \in \Lambda \mid \omega, d \omega \in \mathcal{H}_{g}\right\} .
$$

Let $\delta$ be its formal adjoint, again viewed as unbounded operator, with domain

$$
\operatorname{dom}(\delta):=\left\{\omega \in \Lambda \mid \omega, \delta \omega \in \mathcal{H}_{g}\right\} .
$$

Denote by $\bar{d}$ and $\bar{\delta}$ the respective closures of $d$ and $\delta$. Thus, the domains of $\bar{d}$ and $\bar{\delta}$ are the maximal domains of $d$, resp. $\delta$ :

$$
\begin{aligned}
& \operatorname{dom}(\bar{d})=\left\{\omega \in \mathcal{H}_{g} \mid \exists \omega_{j} \in \operatorname{dom}(d), \eta \in \mathcal{H}_{g} \text { s.t. } \omega_{j} \rightarrow \omega, d \omega_{j} \rightarrow \eta \text { in } \mathcal{H}_{g}\right\} \\
& \operatorname{dom}(\bar{\delta})=\left\{\omega \in \mathcal{H}_{g} \mid \exists \omega_{j} \in \operatorname{dom}(\delta), \eta \in \mathcal{H}_{g} \text { s.t. } \omega_{j} \rightarrow \omega, \delta \omega_{j} \rightarrow \eta \text { in } \mathcal{H}_{g}\right\} .
\end{aligned}
$$

Although $\bar{d}$ and $\bar{\delta}$ are formal adjoints to each other, in general $\bar{d}^{*}$ and $\bar{\delta}$ may not be equal. By means of an $L^{2}$ version of Stokes' theorem, Cheeger has shown that (an equivalent form of) the vanishing condition (2.1) ensures the equality of the two operators.

We collect in the following statement those 'quasi-isometric invariant' results in $[\mathrm{C} 1, \mathrm{C} 2]$ which are fundamental for the construction of the signature $K$-cycle for Witt spaces.

Theorem 2.1 (Cheeger). Let $X^{n}$ be a closed Witt space, endowed with a piecewise Riemannian metric of conic type. Then:

(i) Stokes' theorem holds in the $L^{2}$ sense, i.e.

$$
<\bar{d} \omega_{1}, \omega_{2}>=<\omega_{1}, \bar{\delta} \omega_{2}>, \quad \forall \omega_{1} \in \operatorname{dom}(\bar{d}), \omega_{2} \in \operatorname{dom}(\bar{\delta})
$$

(ii) $\bar{d}^{*}=\bar{\delta}$;

(iii) the operator $\mathrm{D}_{g}:=\bar{d}+\bar{\delta}$, with domain $\operatorname{dom}\left(\mathrm{D}_{g}\right):=\operatorname{dom}(\bar{d}) \cap \operatorname{dom}(\bar{\delta})$, is selfadjoint;

(iv) the heat operator $\mathrm{e}^{t \mathrm{D}_{g}^{2}}$ is trace class on $\mathcal{H}_{g}$ and there exists $C>0$ such that

$$
\operatorname{Tr}\left(e^{-t \mathrm{D}_{g}^{2}}\right) \leq C t^{-\frac{n}{2}}, \quad \forall t \in(0,1]
$$




\section{Signature $K$-cycles}

In order to recast the above mentioned results in the framework of noncommutative geometry, we need to recall a few basic definitions.

An even p-summable unbounded Fredholm module over $C(X)$, where $p \geq$ 1 , is a pair $(\mathcal{H}, D)$ such that:

(i) the Hilbert space $\mathcal{H}$ is endowed with a unital *-representation of $C(X)$ and $D$ is a self-adjoint unbounded linear operator on $\mathcal{H}$;

(ii) there is a dense *-subalgebra $\mathcal{A} \subset C(X)$ such that $\forall a \in \mathcal{A}$, the domain of $D$ is invariant by $a$ and $[D, a]$ extends to a bounded operator on $\mathcal{H}$;

(iii) $\left(1+D^{2}\right)^{-\frac{1}{2}} \in \mathcal{L}^{p}(\mathcal{H}):=$ the ideal of $p$-Schatten class operators on $\mathcal{H}$;

(iv) $\mathcal{H}$ is equipped with a grading $\tau=\tau^{*}, \tau^{2}=I$, such that $\tau f=f \tau, \forall f \in$ $C(X)$ and $D \tau=-\tau D$.

The corresponding odd notion is obtained by simply omitting the last condition. Such a Fredholm module, also called a $K$ - cycle over $C(X)$, determines a $K$-homology class,

$$
[\mathcal{H}, D] \in K_{*}(X), * \in \mathbb{Z} / 2 \mathbb{Z} \text {. }
$$

At this point we should mention that, on an even-dimensional Riemannian Witt space $\left(X^{2 n}, g\right)$, the 'signature grading' operator is defined as in the smooth case, by first considering the corresponding duality operator

$$
*_{p}: L_{2}\left(\Lambda^{p} M\right) \longrightarrow L_{2}\left(\Lambda^{2 n-p} M\right) \text {, }
$$

then turning it into an involution by setting:

$$
\tau_{g}=i^{p(p-1)+n} *_{p} \text { on } L_{2}\left(\Lambda^{p} M\right) .
$$

Given a Fredholm module $(\mathcal{H}, \mathrm{D})$ over $C(X)$, one defines the support of an operator $A$ on $\mathcal{H}$, denoted $\operatorname{Supp}(A)$, as the complement of the union of the open sets $U \times V \subset X \times X$ such that

$$
\varphi_{1} A \varphi_{2}=0, \quad \forall \varphi_{1} \in C_{c}(U), \varphi_{2} \in C_{c}(V)
$$


If $X$ is a metric space with distance function $\rho$, we shall say that $(\mathcal{H}, D)$ over $C(X)$ has the unit propagation speed property if the corresponding wave operator satisfies

$$
\operatorname{Supp}\left(\mathrm{e}^{i t D}\right) \subset\{(x, y) \in X \times X|\rho(x, y) \leq| t \mid\} .
$$

With these explanations, we may now define and state the basic properties of the signature $K$-cycle on a Witt space.

Theorem 3.1. Let $X^{n}$ be a closed Witt space, equipped with a piecewise Riemannian metric of conic type. Then:

(i) the pair $\left(\mathcal{H}_{g}, D_{g}\right)$ defines a p-summable unbounded Fredholm module over $C(X)$, for any $p>\operatorname{dim}(X)$, and therefore a $K$-homology class $\left[\mathcal{H}_{g}, \mathrm{D}_{g}\right] \in K_{*}(X), *=n(\bmod 2)$;

(ii) the class

$$
\sigma_{X}=\left[\mathcal{H}_{g}, \mathrm{D}_{g}\right]
$$

is independent of the choice of the piecewise Riemannian metric of conic type $g$;

(iii) $\left(\mathcal{H}_{g}, \mathrm{D}_{g}\right)$ has the unit propagation speed property;

(iv) if $M$ is a closed smooth manifold, one has

$$
\sigma_{X \times M}=\sigma_{X} \otimes \sigma_{M}
$$

Proof: (i) As dense *-subalgebra $\mathcal{A} \subset C(X)$, one can take the subalgebra $C^{L i p}(X)$ of Lipschitz functions with respect to the given metric structure on $X$. Indeed, if $f \in C^{\text {Lip }}(X)$ then

$$
f \cdot \operatorname{dom}\left(\mathrm{D}_{g}\right) \subset \operatorname{dom}\left(\mathrm{D}_{g}\right) .
$$

Moreover the commutator $\left[\mathrm{D}_{g}, f\right]$ is given by the usual Clifford multiplication (on forms) by $d f$, therefore extends to a bounded operator on $\mathcal{H}_{g}$. The fact that

$$
\operatorname{Tr}\left(\left(1+D_{g}^{2}\right)^{-p}\right)<\infty, \quad \forall p>\operatorname{dim}(X)
$$

is an easy consequence of (2.2). 
(ii) The independence of the metric follows from the homotopy invariance of $K$-homology, along similar lines of reasoning as in [H1, Section 6].

(iii) Hilsum's scheme in [H2, Section 1] for proving the finite propagation property in the case of Lipschitz manifolds also applies to our setting to give (3.1).

(iv) Finally, the multiplicativity property (3.2) becomes obvious when we take the Riemannian structure on $X \times M$ to be the direct product of a Riemannian metric of conic type on $X$ by any (smooth) Riemannian metric on $M$.

Remark 3.2. It follows from Cheeger's combinatorial definition of the $L$ classes for Witt spaces [C2, Section 9] that

$$
c h_{*}\left(\sigma_{X} \otimes \mathbb{Q}\right)=c h_{*}\left(\Delta_{X} \otimes \mathbb{Q}\right) \text { in } H_{*}(X ; \mathbb{Q}),
$$

where $\Delta_{X} \in K_{*}(X) \otimes \mathbb{Z}[1 / 2]$ is the Sullivan-Siegel [Si] $K$-orientation class of the Witt space $X$ and $c h_{*}: K_{*}(X) \otimes \mathbb{Q} \rightarrow H_{*}(X ; \mathbb{Q})$ denotes the usual homological Chern character. Therefore, over $\mathbb{Q}$, the signature class $\sigma_{X}$ defined above coincides with the $K$-orientation class $\Delta_{X}$ :

$$
\sigma_{X} \otimes \mathbb{Q}=\Delta_{X} \otimes \mathbb{Q} \text { in } K_{*}(X) \otimes \mathbb{Q}
$$

\section{Straight Chern character}

We now recall, following [MW1], the construction of the straight Chern character in Alexander-Spanier homology (cf. [CM]).

First, the Alexander-Spanier homology of a compact separable Haussdorf space $X$ is defined as follows. Let $\mathcal{C}(X)$ denote the collection of all finite open covers of $X$. Given $\mathcal{U} \in \mathcal{C}(X)$, let $C_{y}(X ; \mathcal{U})$ be the space of (Borel) measures on $X^{q+1}$ which are compactly supported with their supports contained in the subset $\mathcal{U}^{q+1}:=\cup_{U \in \mathcal{U}} U^{q+1}$ of $X^{q+1}$. The boundary operator $\partial: C_{q}(X ; \mathcal{U}) \longrightarrow$ $C_{q-1}(X ; \mathcal{U})$ is given by the formula

$$
(\partial \mu)(\varphi):=\mu(\delta(\varphi)), \quad \forall \mu \in C_{q}(X ; \mathcal{U}), \forall \varphi \in C\left(X^{q}\right),
$$

where $\delta$ is the Alexander-Spanier coboundary given by

$$
(\delta \varphi)\left(x_{0}, x_{1}, \cdots, x_{q}\right):=\sum_{i=0}^{q}(-1)^{i} \varphi\left(x_{0}, \cdots, \hat{x}_{i}, \cdots, x_{q}\right) .
$$


Let $\bar{H}_{*}(X ; \mathcal{U})$ denote the homology of the chain complex $C_{q}(X ; \mathcal{U})$. Now if $\mathcal{V}$ is a refinement of $\mathcal{U}$, the natural chain homomorphism given by the inclusion $\epsilon_{\mathcal{U}}^{\mathcal{V}}: C_{*}(X ; \mathcal{V}) \longrightarrow C_{*}(X ; \mathcal{U})$, induces a homomorphism:

$$
\left(\epsilon_{\mathcal{U}}^{\mathcal{V}}\right)_{*}: \bar{H}_{*}(X ; \mathcal{V}) \longrightarrow \bar{H}_{*}(X ; \mathcal{U})
$$

- Thus the collection of groups $\left\{\bar{H}_{*}(X ; \mathcal{U})\right\}_{\mathcal{U} \in \mathcal{C}(X)}$ forms an inverse system of abelian groups. The Alexander-Spanier homology of $X$ is the inverse limit of the inverse system $\left\{\bar{H}_{*}(X ; \mathcal{U})\right\}_{\mathcal{U} \in \mathcal{C}(X)}$ :

$$
\bar{H}_{*}(X ; \mathbb{C}):=\lim _{\leftarrow} \bar{H}_{*}(X ; \mathcal{U})
$$

Thus, a class $[\mu] \in \bar{H}_{q}(X ; \mathbb{C})$ is represented by a family $\left\{\mu_{\mathcal{U}}\right\}_{U \in \mathcal{C}(X)}$, where each $\mu_{\mathcal{U}}$ is a cycle in $C_{q}(X ; \mathcal{U})$ and such that $\mu_{\mathcal{U}}$ and $\mu_{\mathcal{V}}$ are homologous in $C_{q}(X ; \mathcal{U})$ whenever $\mathcal{V}$ is a refinement of $\mathcal{U}$.

We proceed now with the definition of the straight Chern character. Let $\mathfrak{E}_{i}^{p}(\mathcal{A}), i \in \mathbb{Z}_{2}$, denote the collection of all (even, resp. odd) unbounded $K$ cycles over $X$, which are $p$-summable with respect to a fixed $*$-subalgebra $\mathcal{A} \subset C(X)$ as in the previous section; in addition, we assume that $\mathcal{A}$ contains partitions of unity subordinated to any finite open cover of $X$. These sets form Abelian semigroups under the direct sum operation. To turn them into groups, appropriate identifications are required. The equivalence relation suitable for our purposes is generated by special homotopies and addition of degenerate elements.

A special homotopy in $\mathfrak{E}_{i}^{p}(\mathcal{A})$ is a family $\left(\mathcal{H}_{s}, D_{s}\right) \in \mathfrak{E}_{i}^{p}(\mathcal{A}), s \in[0,1]$, such that:

(i) all Hilbert spaces $\mathcal{H}_{s}$ share the same underlying topological vector space $\mathcal{H}$ and the same module structure over $C(X) ;$

(ii) $[0,1] \ni s \mapsto \gamma_{s} \in \mathcal{L}(\mathcal{H})$ is norm continuous;

(iii) for any $f \in \mathcal{A}$, the map $[0,1] \ni s \mapsto\left[D_{s}, f\right] \in \mathcal{L}(\mathcal{H})$ is piecewise $C^{\mathbf{1}}$ in the norm topology;

(iv) the map $[0,1] \ni s \mapsto\left(1+D_{s}^{2}\right)^{-\frac{1}{2}} \in \mathcal{L}^{p}(\mathcal{H})$ is piecewise $C^{1}$ in the norm topology and continuous in the $\mathcal{L}^{p}$-norm topology;

(v) in the even case, the map $[0,1] \ni s \mapsto \gamma_{s} \in \mathcal{L}(\mathcal{H})$ is norm continuous. 
A $K$-cycle $(\mathcal{H}, D)$ with $D$ invertible and $[D, a]=0, \forall a \in \mathcal{A}$, will be called degenerate.

We now declare that two $K$-cycles $\left(\mathcal{H}_{0}, D_{0}\right)$ and $\left(\mathcal{H}_{1}, D_{1}\right)$ are equivalent, and write $\left(\mathcal{H}_{0}, D_{0}\right) \sim\left(\mathcal{H}_{1}, D_{1}\right)$, if there exists a degenerate $K$-cycle $\left(\mathcal{H}_{d}, D_{d}\right)$ and a special homotopy $\left(\widetilde{\mathcal{H}}_{s}, \widetilde{D}_{s}\right), s \in[0,1]$, such that $\left(\widetilde{\mathcal{H}}_{i}, \widetilde{D}_{i}\right)$ is unitarily equivalent to $\left(\mathcal{H}_{i} \oplus \mathcal{H}_{d}, D_{i} \oplus D_{d}\right)$, for $i=0,1$.

We now proceed to define the character class of an even $K$-cycle $(\mathcal{H}, D) \in$ $\mathfrak{E}_{0}^{p}(\mathcal{A})$, as an element in the Alexander-Spanier homology group $\bar{H}_{*}(X ; \mathbb{C})$. More precisely, for each finite cover $\mathcal{U}=\left\{U_{\alpha}\right\}$ of $X$, we shall construct an Alexander-Spanier $\mathcal{U}$-cycle $\chi\left(\mathcal{H}, D ; P_{\mathcal{L}}\right) \in \bar{H}_{*}(X ; \mathbb{C})$, such that if $\mathcal{U}^{\prime}$ is a refinement of $\mathcal{U}$ then $\chi\left(\mathcal{H}, D ; P_{\mathcal{U}^{\prime}}\right)$ and $\chi\left(\mathcal{H}, D ; P_{\mathcal{U}}\right)$ are homologous in $C_{*}(X ; \mathcal{U})$

To begin with, consider a parametrix $Q$ of $D^{+}$such that

$$
S^{+}:=I^{+}-Q D^{+} \in \mathcal{L}^{1}\left(\mathcal{H}^{+}\right), S^{-}:=I-D^{+} Q \in \mathcal{L}^{1}\left(\mathcal{H}^{-}\right)
$$

its existence is ensured by the very definition of a finitely summable $K$-cycle. Given a finite cover $\mathcal{V}=\left\{V_{\alpha}\right\}$ of $X$, we can $\mathcal{V}$-localize both $D$ and $Q$ by choosing a partition of unity $\left\{\varphi_{\alpha}\right\} \subset \mathcal{A}$ subordinated to $\mathcal{V}$ and replacing $T=D$ or $Q$ by

$$
T_{\mathcal{V}}=\sum_{\varphi_{\boldsymbol{\alpha}} \varphi_{\beta} \neq 0} \varphi_{\alpha} T \varphi_{\beta}
$$

the operator thus obtained is clearly supported inside $\mathcal{V}^{2} \subset X \times X$. The corresponding remainders $S_{\mathcal{V}}^{+}, S_{\mathcal{V}}^{-} \in \mathcal{L}^{1}$ will therefore be supported inside $(S t \mathcal{V})^{2}$, where $S t \mathcal{V}=\left\{S t V_{\alpha}\right\}$ and

$$
S t V_{\alpha}=\cup\left\{V \in \mathcal{V}, V \cap V_{\alpha} \neq \emptyset\right\} \text {. }
$$

Fix now a finite cover $\mathcal{U}=\left\{U_{\alpha}\right\}$ and choose $\mathcal{V}$ such that $S t^{2 q+6} \mathcal{V}$ is a refinement of $\mathcal{U}$. Consider the operator $L_{\mathcal{V}}$ with inverse $L_{\mathcal{V}}^{-1}$ given by

$$
L_{\mathcal{V}}=\left(\begin{array}{cc}
S_{\mathcal{V}}^{+} & -\left(I+S_{\mathcal{V}}^{+}\right) Q_{\mathcal{V}} \\
D_{\mathcal{V}}^{+} & S_{\mathcal{V}}^{-}
\end{array}\right), \quad L_{\mathcal{V}}^{-1}=\left(\begin{array}{cc}
S_{\mathcal{V}}^{+} & Q_{\mathcal{V}}\left(I+S_{\mathcal{V}}^{-}\right) \\
-D_{\mathcal{V}}^{+} & S_{\mathcal{V}}^{-}
\end{array}\right),
$$

and form the idempotent

$$
P_{\mathcal{U}}=L_{\mathcal{V}}\left(\begin{array}{cc}
I^{+} & 0 \\
0 & 0
\end{array}\right) L_{\mathcal{V}}^{-1}=\left(\begin{array}{cc}
\left(S_{\mathcal{V}}^{+}\right)^{2} & S_{\mathcal{V}}^{+}\left(I+S_{\mathcal{V}}^{+}\right) Q_{\mathcal{V}} \\
S_{\mathcal{V}}^{-} D_{\mathcal{V}}^{+} & I-\left(S_{\mathcal{V}}^{-}\right)^{2}
\end{array}\right),
$$

supported inside the subset $\left(S t^{4} \mathcal{V}\right)^{2}$. 
One now defines the Alexander-Spanier $\mathcal{U}$-cycle $\chi_{2 q}\left(\mathcal{H}, D ; P_{\mathcal{U}}\right)$ as the $x$ tisymmetric measure given by the formula

$$
\begin{aligned}
& \chi_{2 q}\left(\mathcal{H}, F ; P_{\mathcal{U}}\right)\left(f^{0} \otimes f^{1} \otimes \cdots \otimes f^{2 q}\right) \\
& \quad:=\frac{(2 \pi i)^{q}}{q !(2 q+1)} \sum_{\sigma \in \mathfrak{S}_{2 q+1}} \operatorname{sign}(\sigma) \operatorname{Tr}\left(P_{\mathcal{U}} f^{\sigma(0)} P_{\mathcal{U}} f^{\sigma(1)} \cdots P_{\mathcal{U}} f^{\sigma(2 q)}\right),
\end{aligned}
$$

for any $f^{0}, f^{1}, \ldots, f^{2 q} \in C(X)$, where $\mathfrak{S}_{2 q+1}$ is the permutation group of the set $\{0,1,2, \ldots, 2 q\}$. Equivalently (see [MW1, Lemma 1.8]),

$$
\begin{aligned}
& \chi_{2 q}\left(\mathcal{H}, D ; P_{\mathcal{U}}\right)\left(f^{0} \otimes f^{1} \otimes \cdots \otimes f^{2 q}\right) \\
& \quad=\frac{(2 \pi i)^{q}}{q !} \sum_{\sigma \in \mathbb{S}_{2 q}} \operatorname{sign}(\sigma) \operatorname{Tr}\left(P_{\mathcal{U}} f^{0}\left[P_{\mathcal{U}}, f^{\sigma(1)}\right] \cdots\left[P_{\mathcal{U}}, f^{\sigma(2 q)}\right]\right) .
\end{aligned}
$$

One can easily check that $\chi_{2 q}\left(\mathcal{H}, D ; P_{\mathcal{U}}\right)$ is indeed supported in $\mathcal{U}^{2 q+1}$ and that it is a cycle. Thus,

$$
\left[\chi_{2 q}\left(\mathcal{H}, D ; P_{\mathcal{U}}\right)\right] \in \bar{H}_{2 q}(X ; \mathcal{U})
$$

One then gets a global Alexander-Spanier class, $\overline{c h}_{2 q}(\mathcal{H}, \mathrm{D})$, by taking the inverse limit:

$$
\overline{c h}_{2 q}(\mathcal{H}, \mathrm{D}):=\lim _{\leftarrow}\left\{\left[\chi_{2 q}\left(\mathcal{H}, D ; P_{\mathcal{U}}\right)\right]\right\}_{\mathcal{U} \in \mathcal{C}(X)} \in \bar{H}_{2 q}(X ; \mathbb{C})
$$

Finally, the (total) character class of the $K$-cycle $(\mathcal{H}, D)$ is defined as

$$
\overline{c h}_{*}(\mathcal{H}, \mathrm{D}):=\prod_{q \geq 0} \overline{c h}_{2 q}(\mathcal{H}, \mathrm{D}) \in \prod_{q \geq 0} \bar{H}_{2 q}(X ; \mathbb{C})
$$

The class $\overline{c h}_{*}(\mathcal{H}, \mathrm{D})$ is independent of the choices made in the above definition. Moreover, the character construction factors through the equivalence relation:

$$
\left(\mathcal{H}_{1}, D_{1}\right) \sim\left(\mathcal{H}_{2}, D_{2}\right) \Longrightarrow \overline{c h}_{*}\left(\mathcal{H}_{1}, D_{1}\right)=\overline{c h}_{*}\left(\mathcal{H}_{2}, D_{2}\right)
$$

Having established the unit propagation speed property of the signature $K$-cycle $\left(\mathcal{H}_{g}, \mathrm{D}_{g}\right)$ in Theorem 3.1 , we can express its straight character in terms of 'propagation cycles', as in [MW2]. Let us recall this construction. 
Since the metric $g$ is kept fixed, we may drop the corresponding subscript from the riotation.

Let $\bar{u} \in C^{\infty}(\mathbb{R})$ be an even function such that the function $\bar{v}(x)=1-$ $x^{2} \bar{u}(x)$ is Schwartz and both $\bar{u}$ and $\bar{v}$ have Fourier transforms supported in $\left(-\frac{1}{4}, \frac{1}{4}\right)$. Since $\bar{u}$ and $\bar{v}$ are even, there are smooth functions $u, v$, such that

$$
u\left(x^{2}\right)=\bar{u}(x), \quad v\left(x^{2}\right)=\bar{v}(x) .
$$

Note that $v$ is again Schwartz and so is the function

$$
w(x)=v(x)(1+v(x)) u(x)=\frac{1-v(x)^{2}}{x} v(x) .
$$

We form the idempotent

$$
P(t \mathrm{D})=\left(\begin{array}{cc}
\left(v\left(t^{2} \mathrm{D}^{2}\right)\right)^{2} \gamma & w\left(t^{2} \mathrm{D}^{2}\right) \cdot t \mathrm{D} \gamma \\
-v\left(t^{2} \mathrm{D}^{2}\right) \cdot t \mathrm{D} \gamma & \left(v\left(t^{2} \mathrm{D}^{2}\right)\right)^{2} \gamma
\end{array}\right)+\left(\begin{array}{cc}
\frac{1-\gamma}{2} & 0 \\
0 & \frac{1-\gamma}{2}
\end{array}\right)
$$

and then define an Alexander-Spanier cycle $\Lambda_{*}(\mathcal{H}, t \mathrm{D})$ by setting

$$
\begin{aligned}
& \Lambda_{2 q}(\mathcal{H}, t \mathrm{D})\left(f^{0} \otimes f^{1} \otimes \cdots \otimes f^{2 q}\right) \\
& \quad:=\frac{(2 \pi i)^{q}}{q !(2 q+1) 2} \operatorname{Tr}\left(\sum_{\sigma \in \mathfrak{S}_{2 q+1}} \operatorname{sign}(\sigma) P(t \mathrm{D}) f^{\sigma(0)} \cdots P(t \mathrm{D}) f^{\sigma(2 q)}\right) ;
\end{aligned}
$$

here $f^{0}, \ldots, f^{2 q} \in C(X), q>0$ and $\mathfrak{S}_{2 q+1}$ is the permutation group of the set $\{0,1, \ldots, 2 q\}$; for $q=0$,

$$
\Lambda_{0}(\mathcal{H}, t \mathrm{D})\left(f^{0}\right):=\frac{1}{2} \operatorname{Tr}\left(P(t \mathrm{D}) f^{0}-\left(\begin{array}{cc}
\frac{1-\gamma}{2} & 0 \\
0 & \frac{1-\gamma}{2}
\end{array}\right) f^{0}\right) .
$$

Thus, $\Lambda_{*}(\mathcal{H}, t \mathrm{D})$ is a skew-symmetric measure on $X^{\times(2 q+1)}=X \times \cdots \times X$ $\left(2 q+1\right.$ times), supported in the $|t|$-neighborhood of the diagonal in $X^{\times(2 q+1)}$. It was shown in [MW2] that the family

$$
\Lambda_{*}\{\mathcal{H}, \mathrm{D}\}:=\left\{\Lambda_{*}(\mathcal{H}, t \mathrm{D})\right\}_{t>0}
$$

defines an Alexander-Spanier cycle which, moreover, represents the homology class $\overline{c h}_{*}(\mathcal{H}, \mathrm{D})$.

By Theorem 3.1 (ii), the K-homology class of $(\mathcal{H}, \mathrm{D})$ is independent of the choice of the metric. It is not automatic, but still true, that the AlexanderSpanier class $\overline{c h}_{*}(\mathcal{H}, \mathrm{D})$ is also independent of the chosen metric. 
Proposition 4.1 (Definition). The Alexander-Spanier homology class

$$
\overline{c h}_{*}\left(\sigma_{X}\right)=\overline{c h}_{*}\left(\mathcal{H}_{g}, \mathrm{D}_{g}\right)
$$

does not depend on the choice of the piecewise Ricmannian metric of conic type g.

Proof: If $g_{0}$ and $g_{1}$ are two such metrics on $X$, let

$$
g_{s}=(1-s) g_{0}+s g_{1}, \quad \forall s \in[0,1] .
$$

One can check that that the family of $K$-cycles $\left(\mathcal{H}_{s}, \mathrm{D}_{s}\right)$ forms a special homotopy in $\mathfrak{E}_{*}^{p}\left(C^{\text {Lip }}(X)\right)$. Thus, the $K$-cycles $\left(\mathcal{H}_{0}, \mathrm{D}_{0}\right)$ and $\left(\mathcal{H}_{1}, \mathrm{D}_{1}\right)$ are equivalent.

For our purposes here, the key feature of the straight Chern character is its naturality with respect to open embeddings as well as taking products by smooth manifolds.

Lemma 4.2 (Locality). Let $(U, g) \hookrightarrow\left(X_{i}, g_{i}\right), i=1,2$, be two open embeddings of Riemannian Witt spaces and $K \subset U$ a compact subset. Then, for $t>0$ sufficiently small and $\forall f^{0}, \ldots, f^{2 q} \in C(U)$ with $\operatorname{Supp}\left(f^{k}\right) \subset K$, one has

$$
\begin{aligned}
& \Lambda_{2 q}\left(\mathcal{H}_{1}, t \mathrm{D}_{1}\right)\left(f^{0} \otimes f^{1} \otimes \cdots \otimes f^{2 q}\right) \\
& \quad=\Lambda_{2 q}\left(\mathcal{H}_{2}, t \mathrm{D}_{2}\right)\left(f^{0} \otimes f^{1} \otimes \cdots \otimes f^{2 q}\right)
\end{aligned}
$$

here $\left(\mathcal{H}_{i}, D_{i}\right)$ stands for the signature $K$-cycle of $X_{i}$ with respect to a piecewise Riemannian metric of conic type $g_{i}, i=1,2$.

Proof: It is virtually identical to the proof of [MW2, Theorem 2.2].

Lemma 4.3 (Multiplicativity). Let $X=M^{2 q} \times W^{4 l}$, where $M$ is a compact smooth manifold and $W$ is a compact Witt space, and denote by $\pi$ the projection on the first factor. Then for any smooth Alexander-Spanier 2qcocycle $\varphi$ on $M$, one has

$$
<\overline{c h}_{2 q}\left(\sigma_{X}\right),\left[\pi^{*}(\varphi)\right]>=\operatorname{index}\left(D_{W}\right) 2^{q}<\mathcal{L}(M) \cap[M],[\varphi]>,
$$

where $D_{W}$ stands for a Cheeger signature opcrator $D_{W}$ on $W$ and $\mathcal{L}(M)$ denotes the Atiyah-Hirzebruch $\mathcal{L}$-class of $M$. 
Proof: Choosing a suitable product metric on $X$, we can argue as in the proof of the product formula in [MW3, Proposition 5.1] to get

$$
<\overline{c h}_{2 q}\left(\sigma_{X}\right),\left[\pi^{*}(\varphi)\right]>=\operatorname{index}\left(D_{W}\right)<\overline{c h}_{2 q}\left(\sigma_{M}\right),[\varphi]>
$$

But in the case of a smooth manifold, the straight Chern character gives (cf. [MW2, Theorem 3.4]):

$$
\overline{c h}_{*}\left(\sigma_{M}\right)=2^{q} \mathcal{L}(M) \cap[M] .
$$

\section{Local formulae for $L$-classes}

In this section we shall relate the straight character of a signature $K$-cycle on a Witt space to the Goresky-MacPherson-Siegel homology $L$-class.

We recall (cf. [Si]) that Witt spaces satisfy rational Poincaré duality for the intersection homology groups of middle perversity. More precisely, if $X$ is a closed Witt space, the quadratic form

$$
I H_{k}(X ; \mathbb{Q}) \times I H_{m-k}(X ; \mathbb{Q}) \longrightarrow \mathbb{Q}
$$

is non-degenerate. Furthermore, the signature of this quadratic form is a Witt cobordism invariant.

This property of the intersection homology signature can be used to define the homology $L$-class of a Witt space (valued in the ordinary homology) using transversality (cf. $[\mathrm{GM}]$ ). To fix the ideas, we restrict our attention to the case when $X$ is of even dimension.

Assume first $2 k>2 n+1,2 n=\operatorname{dim}(X)$. There is a canonical isomorphism

$$
H^{k}(X ; \mathbb{Q}) \cong\left[X, S^{k}\right] \otimes \mathbb{Q}
$$

where $\left[X, S^{k}\right]$ denotes the set of homotopy classes of maps. By transversality, every class $[f]$ can be represented by a map $f: X \rightarrow S^{k}$ which is transverse at the north pole $p \in S^{k}$. This implies that $f^{-1}(p) \subset X$ is itself a Witt space. Set

$$
0_{k}([f]):=\operatorname{sign}\left(f^{-1}(p)\right)
$$

where $\operatorname{sign}\left(f^{-1}(p)\right)$ is the intersection homology signature of $f^{-1}(p)$. This assignment defines a homomorphism

$$
\theta_{k}: H^{k}(X ; \mathbb{Q}) \longrightarrow \mathbb{Z},
$$


which, by duality, determines an (ordinary) homology class:

$$
L_{k}(X):=\theta_{k} \text { in } H_{k}(X ; \mathbb{Q}) \cong \operatorname{Hom}\left(H_{k}(X ; \mathbb{Q}), \mathbb{Q}\right) .
$$

The initial restriction $2 k>2 n+1$ is then removed by setting

$$
L_{k}(X):=L_{k}\left(X \times S^{l}\right), \quad l \text { large, }
$$

the identification being made via the natural inclusion map.

Note that the only non-trivial components of the $L$-class are $L_{2 n-4 q}(X)$, $q \geq 0$. The total homology L-class is, by definition

$$
L(X):=\sum_{0 \leq 4 q \leq 2 n} L_{2 n-4 q}(X) \in H_{*}(X ; \mathbb{Q})
$$

To relate the homology $L$-class with the straight character of the signature $K$-cycle, it is convenient to renormalize it by setting:

$$
\mathcal{L}^{n}(X):=\sum_{0 \leq k \leq n} 2^{q} L_{2 q}(X) \in H_{*}(X ; \mathbb{Q})
$$

equivalently,

$$
\mathcal{L}_{2 q}^{\cap}(X):=2^{q} L_{2 q}(X) .
$$

The class $\mathcal{L}^{\cap}(X)$ will be called the total homology $\mathcal{L}$-class of $X$. Note that, for a PL manifold $M$,

$$
\mathcal{L}^{\cap}(M)=\mathcal{L}(M) \cap[M]
$$

We are now ready to state the main result of this paper.

Theorem 5.1. Let $X^{2 n}$ be a closed Witt space. Then, for any piecewise Riemannian metric of conic type on $X$, the corresponding Alexander-Spanier cycle $\Lambda_{*}\{\mathcal{H}, \mathrm{D}\}$ represents the homology $\mathcal{L}$-class, i.e.

$$
\overline{c h}_{2 q}\left(\sigma_{X}\right)=\mathcal{L}_{2 q}^{\cap}(X) \text { in } H_{2 q}(X ; \mathbb{R}) .
$$

Proof: Retracing the steps of the construction of $L$-classes, we shall first prove equation (5.4) for $2 q>n+\frac{1}{2}$.

Let $f: X \rightarrow S^{2 q}$. Then the homotopy class of $f$ determines a cohomology class $f^{*}(u) \in \bar{H}^{2 q}(X ; \mathbb{R})$, where $u \in \bar{H}^{2 q}\left(S^{2 q} ; \mathbb{R}\right)$ is a generator such that

$$
<\left[S^{2 q}\right],[u]>=1 \text {. }
$$


Explicitly, equation (5.4) means

$$
\Lambda_{2_{q}}\{\mathcal{H}, \mathrm{D}\}\left(f^{*}(u)\right)=2^{q} \theta([f]) .
$$

As both sides are invariant under homotopy of $f$, we can assume that $f$ : $X \rightarrow S^{2 q}$ is transversal and such that

(i) $f^{-1}(p)$ is a Witt space, where $p$ is the north pole of $S^{2 q}$;

(ii) $\exists$ a neighborhood $U \subset S^{2 q}$ of $p$ such that $f^{-1}(U)=U \times f^{-1}(p)$ and $f$ is the projection to $U$ when restricted to $f^{-1}(U)$.

Now represent the cohomology class $u \in \bar{H}^{2 q}\left(S^{2 q} ; \mathbb{R}\right)$ by an AlexanderSpanier cocycle $\varphi$ supported inside $U$. Then the cohomology class $f^{*}(u)$, represented by $f^{*}(\varphi)$, is supported inside $f^{-1}(U)=U \times f^{-1}(p)$.

One can choose a piecewise Riemannian metric of conic type $g_{1}$ on $S^{2 q} \times$ $f^{-1}(p)$ which coincides with the restriction of the metric of $X$ on a neighborhood of the (compact) support of the cocycle $\varphi$ inside $U \times f^{-1}(p)$. Let $\left(\mathcal{H}_{1}, D_{1}\right)$ be the signature $K$-cycle of $\left(S^{2 q} \times f^{-1}(p), g_{1}\right)$. Then, by the Locality Lemma 4.2, we have

$$
\Lambda_{2 q}\{\mathcal{H}, \mathrm{D}\}\left(f^{*}(u)\right)=\Lambda_{2 q}\left\{\mathcal{H}_{1}, \mathrm{D}_{1}\right\}\left(\pi^{*}(\varphi)\right),
$$

where $\pi: S^{2 q} \times f^{-1}(p) \rightarrow S^{2 q}$ denotes the projection.

Applying now the Multiplicativity Lemma 4.3 to the right hand side of equation (5.6), we get

$$
\begin{aligned}
\Lambda_{2 q} & \{\mathcal{H} . \mathrm{D}\}\left(f^{*}(u)\right)=\Lambda_{2 q}\left\{\mathcal{H}_{1}, \mathrm{D}_{1}\right\}\left(\pi^{*}(\varphi)\right) \\
& =\operatorname{sign}_{2}\left(f^{-1}(p)\right) 2^{q}<\mathcal{L}\left(S^{2 q}\right) \cap\left[S^{2 q}\right],[\varphi]> \\
& =2^{q} \operatorname{sign}_{2}\left(f^{-1}(p)\right)<\left[S^{2 q}\right],[u]> \\
& =2^{q} \operatorname{sign}_{2}\left(f^{-1}(p)\right) .
\end{aligned}
$$

As shown in [C1], the intersection homology groups $I H_{*}(W ; \mathbb{R})$ are dual to the $L^{2}$-cohomology groups of $W$. Therefore, the $L^{2}$-signature (which coincides with the index of the signature operator) is equal to the intersection homology signature.

This proves equation (5.5) when $2 q>n+\frac{1}{2}$. For general $q$, one replaces $X$ by $X \times S^{l}$, with $l$ sufficiently large, and one applies once more Lemma 4.3 . 



\section{References}

[Ci] Cheeger, J. On the Hodge theory of Ricmannian pseudomanifolds, Geometry of the Laplace Operator, Proc. Symposia Pure Math. 36 (1980) $91-146$.

[C2] Cheeger, J., Spectral geometry of singular Riemannian spaces, J. Diff. Geom. 18 (1983), 575-657.

[CM] Connes, A. and Moscovici, II., Cyclic cohomology, the Novikov conjecture, and hyperbolic groups, Topology 29 (1990), No.3, 345-388.

[CST] Connes, A., Sullivan, D., and Teleman, N., Quasiconformal mappings, operators on IIilbert space and local formulae for characteristic classes, Topology 33 (1994), No.4, 663-681.

[GM] Goresky, R. M. and MacPherson, R., Intersection homology theory, Topology 19 (1980), 135-162.

[H1] Hilsum, M., Signature operators on Lipschitz manifolds and unbounded Kasparov bimodules, Lecture Notes in Math. 1132 (1985), 254-288.

[H2] Hilsum, M., Fonctorialité en K'-théorie bivariante pour les variétés Lipschitziennes, K-theory 3 (1989), 401-440.

[MW1] Moscovici, H. and Wu, F-B., Index theory without symbols, $\mathbf{C}^{*}$-algebras: 1943 - 1993, Contemporary Mathematics, no. 167, 305-351.

[MW2] Moscovici, H. and Wu, F-B., Localization of topological Pontryagin classes via finite propagation speed, Geometric and Functional Analysis 4 (1994), $52-92$.

[MW3] Moscovici, H. and Wu, F-B., Pontryagin forms for manifolds with framed singular strata, Geometric and Functional Analysis 5 (1995), 702-728.

[Si] Siegel, P.. Witt spaces: a geometric cycle theory for KO-homology at odd primes, Amer. J. Math. 105 (1983), 1067-1105.

[Su] Sullivan, D. Gcometric topology, Part I, MIT Notes, Cambridge, MA, 1970 .

H. M. : Ohio State University, Columbus, OII 43210, U.S.A. F-B. W. : Kansas State University, Manhattan, KS 66506, U.S.A. 
